\title{
Effect of basal insulin therapy with glargine U300 versus basal-bolus insulin therapy in hospitalized patients with type 2 diabetes. Real-world study from India
}

\section{ABSTRACT}

Background. Basal-bolus insulin therapy is the most widely accepted method of glycemic control in noncritically ill patients with T2DM [1]. In this regimen glargine U100 is the most commonly used basal insulin. American Diabetes Association recommends either basal or basal bolus therapy for patients with type 2 diabetes mellitus admitted in general medical and surgical ward with ideal glycemic target ranging between 140-180 mg/dL by estimating four or 6 hourly capillary blood glucose per day. Insulin glargine U300 is a second generation long acting insulin analogue, which has a prolonged pharmacokinetic /pharmacodynamic profile compared to insulin glargine $U 100$ resulting in glucose lowering activity exceeding 24 hours.

Aim. To assess the efficacy and safety of basal insulin regimen (glargine U300) compared to a basal-bolus insulin regimen in patients with type 2 diabetes.

Methods. A prospective single centred parallel group study comparing the efficacy and safety of basal insulin regimen with basal-bolus insulin regimen. A total of 60 patients with type 2 diabetes mellitus admitted in general medical and surgical ward for elective surgery and medical emergency were randomized to either of

Address for correspondence:

Asis Mitra

Ruby General Hospital, Ruby General Hospital Kasba Golpark,

700107 Kolkata, India

e-mail: asis.mitra10@gmail.com

Clinical Diabetology 2021, 10; 2: 180-187

DOI: 10.5603/DK.a2021.0011

Received: 12.10.2020

Accepted: 28.12 .2020 the two regimens. The baseline glycemic parameters were assessed by capillary blood glucose testing thrice before meal and at bedtime with a target capillary blood glucose (CBG) between 140-180 mg/dL. All patients were followed up for seven days.

Results. The number of CBG readings within the target range were higher in basal insulin monotherapy (glargine U300) compared to basal-bolus regimen using (glargine U100 and regular human insulin) which was statistically significant. The incidence of hypoglycemia was lower in the basal insulin regimen. Fewer units of insulin were required in the basal insulin regimen with lower glycemic variability as compared to basalbolus regimen.

Conclusion. Glargine $\mathbf{U} \mathbf{3 0 0}$ monotherapy as a basal insulin is non-inferior to basal- bolus therapy in noncritically ill hospitalised patients in glycemic control, with fewer incidences of hypoglycemia, less amount of insulin requirement to achieve target capillary blood glucose level and lower glycemic variability. (Clin Diabetol 2021; 10; 2: 180-187)

Key words: type 2 diabetes, Glargine U 300, general hospital ward, basal insulin, basal bolus insulin

\section{Introduction}

People with diabetes are more likely to be hospitalized and to have longer durations of hospital stay than those without diabetes. People with diagnosed diabetes incur average medical expenditures of $\sim \$ 16,750$ per year, of which $\$ 9,600$ is attributed to diabetes. People 
with diagnosed diabetes, on average, have medical expenditures 2.3 times higher than it would be in the absence of diabetes [2]. Inpatient hyperglycemia is common and associated with poor outcomes including prolonged hospital stay, increased risk of hospital complications and disability after hospital discharge and death [3]. Hyperglycemia is frequently encountered during parenteral (PN) and/or enteral (EN) nutrition in hospitalized patients with and without pre-existing diabetes $[4,5]$. Indeed, it is estimated that more than $50 \%$ of patients on $\mathrm{PN}$ and $30 \%$ of patients on EN experience hyperglycemia whilst in the hospital [6, 7]. Hyperglycemia arises in these patients due to one or more of the following factors: a) diminished insulin sensitivity due to inflammation, stress hormones, and sedentarism [8]; b) increased carbohydrate provision; and c) side-effects of medication such as glucocorticoids that interfere with glucose metabolism [9]. In patients totally reliant on PN, these factors are compounded by the loss of the physiological incretin effect on insulin release, as occurs when entirely bypassing the gastrointestinal tract with intravenous nutrient supply [10]. Furthermore, the diminished glucose-stimulated insulin secretion in diabetic patients with some residual beta-cell function increases their requirement for exogenous insulin. The results of randomized clinical trials in general medicine and surgery patients with T2DM have shown that the use of basal bolus insulin regimens result in better glycemic control and lower frequency of a composite of hospital complications including postoperative wound infection, pneumonia, bacteraemia, and acute renal and respiratory failure compared to treatment with sliding scale regular insulin [11]. Despite these benefits of basal bolus regimen, many physicians are reluctant to integrate basal insulin regimens into their clinical practice possibly due to the fear of hypoglycemia and general clinical inertia [12].

Glycemic targets in hospitalised patients as laid down by ADA 2020 are as follows:

- Insulin therapy should be initiated for treatment of persistent hyperglycemia starting at a threshold $\geq 180 \mathrm{mg} / \mathrm{dL}$ (10.0 mmol/L). Once insulin therapy is started, a target glucose range of 140-180 $\mathrm{mg} / \mathrm{dL}(7.8-10.0 \mathrm{mmol} / \mathrm{L})$ is recommended for the majority of critically ill patients and noncritically ill patients.

- More stringent goals, such as $110-140 \mathrm{mg} / \mathrm{dL}$ (6.1-7.8 $\mathrm{mmol} / \mathrm{L})$, may be appropriate for selected patients if they can be achieved without significant hypoglycemia [13].

Hypoglycemia in hospitalized patients is categorized by blood glucose concentration and clinical correlates: level 1 hypoglycemia is a glucose concentration
54-70 mg/dL (3.0-3.9 mmol/L). Level 2 hypoglycemia is a blood glucose concentration $<54 \mathrm{mg} / \mathrm{dL}(3.0$ $\mathrm{mmol} / \mathrm{L})$, which is typically the threshold for neuroglycopenic symptoms. Level 3 hypoglycemia is a clinical event characterized by altered mental and/or physical functioning that requires assistance from another person for recovery. Levels 2 and 3 require immediate correction of low blood glucose.

Basal insulin or a basal plus bolus correction insulin regimen is the preferred treatment for non-critically ill hospitalized patients with poor oral intake or those who are taking nothing by mouth. An insulin regimen with basal, prandial, and correction components is the preferred treatment for non-critically ill hospitalized patients with good nutritional intake. Use of only a sliding scale insulin regimen in the inpatient hospital setting is strongly discouraged [13].

There are very few studies available comparing glargine U100 and glargine U300 as basal insulin in hospital setting. In a study with glargine U300 in inpatient management resulted in similar glycemic control compared with glargine $\mathrm{U} 100$ and may be associated with a lower incidence of clinically significant hypoglycemia [14].

Objective of present study was to evaluate the efficacy of glargine U300 as monotherapy as basal insulin when compared with basal- bolus insulin regime using glargine $\mathrm{U} 100$ as basal and regular human insulin as bolus insulin.

\section{Materials and methods}

This was a single centred, prospective parallel group randomized study to compare the efficacy of basal insulin with basal bolus insulin in hospitalized patients in medical and surgical wards. Study period was from February 2019 to January 2020. Patients recruitment started post ethical committee approval. A total of 60 patients were included after fulfilling inclusion criteria. Informed Consent was taken from each patient and patients enrolled on basal insulin groups were explained about new therapy and properly counselled. Patients were randomized to either of the two groups. One group received insulin glargine U300 as basal insulin only and other group received glargine U100 as basal-plus regular human insulin as bolus or correctional insulin regime according to hospital protocol. Patients capillary blood glucose (CBG) was assessed 4 times daily (pre-meal and at bedtime) and SOS for symptoms of hypoglycemia, which is the only glycemic parameter used for this study and 4 point CBG assessed in both the groups from day 1 to day 7. Premeal blood glucose testing done within 30 minutes of the start of a meal, with pre-meal, rapid-acting insulin 
administered within 10 minutes before or after the start of the meal.

Objective of this comparative study between the two groups of insulin therapy in hospitalized non critically ill patients were:

- Change in mean daily capillary blood glucose concentration from day one to day seven.

- Number of capillary blood glucose readings between 140 to $180 \mathrm{mg} / \mathrm{dL}$ in both the groups for all 4 readings.

- Number of capillary blood glucose readings between 110 to $140 \mathrm{mg} / \mathrm{dL}$ in both the groups for all 4 readings.

- Number of hypoglycemia - capillary blood glucose level less than $70 \mathrm{mg} / \mathrm{dL}$ (level 1) in both the groups.

- Number of clinically significant hypoglycemia capillary blood glucose level less than $54 \mathrm{mg} / \mathrm{dL}$ (level 2) in both the groups.

- Daily insulin requirements at beginning and end of the study in both the groups.

- Assessment of glycemic variability in both the groups. Inclusion criteria:

- Patients with T2DM requiring admission in general medicine ward.

- Patients with type 2 DM admitted for elective surgery in surgical ward.

- Age group of patients: 30 years to 65 years Exclusion criteria:

- Patients with type 1 DM.

- Gestational diabetes mellitus.

- Patients requiring emergency surgery.

- Patients having past history of diabetic ketoacidosis and non ketotic hyperosmolar state.

- Patients admitted to critical care units.

- Patients requiring nothing per oral (NPO) for 12 hours or more.

- Patients requiring total parenteral nutrition.

- Patients already diagnosed with chronic kidney disease stage 4 and 5 and patients undergoing renal replacement therapy.

\section{Protocol of insulin administration}

All of the patients recruited were either receiving oral feed or feed through nasogastric tube every 4 hourly except 12 midnights to 6 am under supervision of a dietician in our hospital. Average diet intake per day per patient was 1600-1800 kcal depending upon height and weight of which $55 \%$ energy from carbohydrate, $35 \%$ from protein and $10 \%$ from fat. Patients on basal insulin group were given single dose of glargine U300 daily at $10 \mathrm{pm}$ with a starting dose of 0.4 units $/ \mathrm{kg}$ body weight and dose was increased 20 percent daily till glycemic goal (fasting CBG less than $140 \mathrm{mg} / \mathrm{dL}$ ) is achieved. Patients on basal- bolus insulin regimen were given glargine $\mathrm{U} 100$ as basal insulin at bedtime in a similar starting an incremental dose as glargine U300. In addition they have got bolus regular human insulin at a starting dos of 0.2 units $/ \mathrm{kg} /$ day with a correctional insulin dose of 2 units every $50 \mathrm{mg} / \mathrm{dL}$ above CBG reading of $150 \mathrm{mg} / \mathrm{dL}$. Daily increment of bolus insulin by 2 units in each dose was adjusted till glycemic goal was (CBG less than $180 \mathrm{mg} / \mathrm{dL}$ ) achieved. Doses of basal insulin were decreased by $50 \%$ in both the groups of patients on the night day before the surgery.

\section{Hypoglycemia management ptotocol}

- CBG < $70 \mathrm{mg} / \mathrm{dL}$ : if patient is able to receive treatment orally, 20 grams of fast-acting carbohydrate was given either as glucose tablets or $6 \mathrm{oz}$. fruit juice. If patient was unable to receive treatment orally , 25 mL D50 IV was administered.

- Blood glucose was checked every 15 minutes and the same procedure was repeated untill CBG is $\geq 100 \mathrm{mg} / \mathrm{dL}$. Basal insulin dose was decreased by $20 \%$.

\section{Statistical analysis}

Statistical analysis was performed using IBM SPSS STATISTICS subscription for Windows. Continuous variables were represented as mean (SD). Categorical variables were represented as number present [N] (\%). Comparison was done between Basal bolus therapy and Basal insulin therapy. Data were analyzed using a univariate and repeated measures general linear model (GLM). Repeated measures GLM was attempted to compare the difference between the two treatments along with effects of the days of treatment and times of administration of treatment. The between subject's effect was the treatments and the within subject effects were the day of administration and time of administration. Test of sphericity was conducted and if significant, Greenhouse-Geisser or Huynh-Feldt test results were used as appropriate. Univariate methods for continuous variables were independent samples T-test or Mann-Whitney U test as appropriate. For categorical variables, Pearson's chi-square test was used. Statistical significance was $p<0.05$.

\section{Results}

A total of 60 patients were enrolled in the study (basal bolus insulin regimen: 30 , basal insulin: 30 ). The mean (SD) age of the population was 63.4 (9.25) and the average BMI was 28.89 (1.64). The average (SD) duration of diabetes was 11.7 (3.86) years and the 
Table 1. Demography profile $(n=60)$

\begin{tabular}{lccc}
\hline & Basal-bolus insulin therapy $(\mathbf{n}=\mathbf{3 0})$ & Basal insulin therapy $(\mathbf{n}=\mathbf{3 0})$ & P-value \\
\hline Duration of diabetes & $11.7(3.861)$ & $11.03(3.672)$ & 0.496 \\
Age & $63.4(9.254)$ & $58.63(10.04)$ & 0.061 \\
$\mathrm{BMI}$ & $28.897(1.6456)$ & $28.74(1.3556)$ & 0.689 \\
$\mathrm{HbA}_{1 \mathrm{c}}$ & $8.6(0.7548)$ & $8.797(0.8576)$ & 0.35 \\
\hline
\end{tabular}

Table 2. Daily change in mean capillary blood glucose concentration from baseline (day 1) to study end (day 7) between the groups (multi-variate analysis)

\begin{tabular}{|c|c|c|c|c|c|c|c|c|c|}
\hline & \multicolumn{4}{|c|}{$\begin{array}{c}\text { Basal-bolus therapy - human actrapid thrice daily } \\
\text { as bolus and glargine U } 100 \text { as basal }(n=30)\end{array}$} & \multicolumn{4}{|c|}{$\begin{array}{l}\text { Basal insulin therapy using glargine U } 300 \\
\text { mono therapy }(n=30)\end{array}$} & \multirow[t]{2}{*}{ P-value* } \\
\hline & 8:00 AM & 1:00 PM & 7:00 PM & Midnight & 8:00 AM & 1:00 PM & 7:00 PM & Midnight & \\
\hline \multirow[t]{2}{*}{ Day 1} & 244.47 & 320.17 & 312.13 & 196.93 & 294.97 & 330.7 & 360.1 & 257.4 & 0.022 \\
\hline & (73.355) & (62.299) & $(56.256)$ & $(45.661)$ & $(86.092)$ & (70.347) & (63.18) & (48.169) & \\
\hline \multirow[t]{2}{*}{ Day 2} & 129.3 & 213.17 & 249.4 & 155.8 & 197.33 & 241.23 & 280 & 181.37 & \\
\hline & $(32.631)$ & $(48.67)$ & $(44.047)$ & $(21.545)$ & (49.56) & $(48.791)$ & $(52.008)$ & (31.372) & \\
\hline \multirow[t]{2}{*}{ Day 3} & 93.43 & 182.4 & 230.83 & 145.43 & 138.63 & 186.6 & 226.77 & 156.73 & \\
\hline & (16.878) & $(21.896)$ & (31.056) & $(20.401)$ & $(23.881)$ & $(36.264)$ & $(43.117)$ & (14.855) & \\
\hline \multirow[t]{2}{*}{ Day 4} & 93.8 & 180.4 & 205.53 & 139.6 & 124.03 & 163.73 & 194.6 & 145.2 & \\
\hline & (17.458) & (33.154) & (35.596) & (24.095) & (20.895) & $(25.842)$ & $(29.967)$ & (19.641) & \\
\hline \multirow[t]{2}{*}{ Day 5} & 88.83 & 181.1 & 206.33 & 139.7 & 126.73 & 165.93 & 196.7 & 146.6 & \\
\hline & (16.499) & (34.666) & (30.96) & $(24.351)$ & (59.738) & $(45.978)$ & (47.496) & (31.383) & \\
\hline \multirow[t]{2}{*}{ Day 6} & 90.53 & 178.43 & 209.57 & 142.23 & 117 & 159 & 184.47 & 138.93 & \\
\hline & $(27.543)$ & $(22.997)$ & (39.54) & (27.784) & $(40.925)$ & $(35.531)$ & (35.124) & (17.162) & \\
\hline \multirow[t]{2}{*}{ Day 7} & 100.8 & 169.3 & 181.77 & 137.67 & 109.93 & 155.9 & 177.73 & 134.1 & \\
\hline & $(25.292)$ & (19.981) & $(30.656)$ & $(19.902)$ & $(29.024)$ & (26.778) & (22.944) & $(21.43)$ & \\
\hline P-value** & $<0.001$ & $<0.001$ & $<0.001$ & $<0.001$ & $<0.001$ & $<0.001$ & $<0.001$ & $<0.001$ & \\
\hline
\end{tabular}

*P-value for between subjects analysis; **P-value for within subjects analysis

mean $\mathrm{HbA} 1 \mathrm{C}$ was 8.6 (0.75). There was no statistical significance between the arms in the demographic profile (Table 1).

The mean change in the daily capillary blood glucose from day 1 to day 7 is tabulated in Table 2 . There was statistically significant reduction in the daily capillary blood glucose in all four time points from day 1 to day 7 within the groups and between the groups. ( $p$ value $<0.05$ )

Table 3 describes the proportion of samples within the stated $C B G$ levels to understand the number of times the CBG levels of patients were within target or having, severe hypoglycemia or hyperglycemia. There were no patients with severe hypoglycemia. However, there were 368 samples in the basal bolus insulin therapy group and 327 in the basal insulin therapy group having hyperglycemia (CBG > $180 \mathrm{mg} / \mathrm{dL}$ ). This difference in samples were statistically significant between the two groups. 299 samples in BIT group achieved target glycemia (CBG between 140-180 mg/dL) compared to 194 samples in BBIT group which is statistically significant. On the other hand 143 samples in BIT group and 140 samples in BBIT group achieved strict glycemic target (CBG between 110-140 mg/dL). However, this difference was not statistically significant.

Patients treated with basal- bolus regime had higher insulin requirement to achieve glycemic goal compared to basal insulin group. This difference in requirement was statistically significant between the treatments and within each treatment group (Table 4).

The glycemic variability in both groups of therapy were plotted that shows a downward trend in both the treatment arms. However, there is a steadier, more stable decline in the BIT group as compared to BBIT group (Figures 1, 2).

\section{Discussions}

Glargine is a human insulin analogue that differs from the endogenous human insulin by a substitution of glycine for asparagine at position A21 and the addi- 
Table 3. Proportion of samples with different levels of capillary blood glucose in both the groups at all four time points

\begin{tabular}{lccc}
\hline $\begin{array}{l}\text { Capillary blood glucose } \\
\text { levels [mg/dL] }\end{array}$ & $\begin{array}{c}\text { Basal-bolus therapy - human actrapid } \\
\text { thrice daily as bolus and glargine U 100 } \\
\text { as basal }\end{array}$ & $\begin{array}{c}\text { Basal insulin therapy using glargine U } 300 \\
\text { mono therapy }\end{array}$ & $\begin{array}{c}\text { P-value } \\
\text { Less than } 54\end{array}$ \\
$54-69$ & 0 & 0 & - \\
$70-109$ & 13 & 1 & $<0.01$ \\
$110-140$ & 140 & 143 & $<0.001$ \\
$140-180$ & 194 & 299 & 0.845 \\
More than 180 & 368 & 327 & 0.001 \\
\hline
\end{tabular}

Table 4. Insulin requirements at baseline (day 1) and study end (day 7) in both the groups

\begin{tabular}{lccc}
\hline & $\begin{array}{c}\text { Basal-bolus therapy - human actrapid } \\
\text { thrice daily as bolus and glargine U 100 } \\
\text { as basal }\end{array}$ & $\begin{array}{c}\text { Basal insulin therapy using glargine U } \\
\mathbf{3 0 0} \text { mono therapy }\end{array}$ & P-value* \\
\hline Starting dose of insulin & $44.27(6.802)$ & $24(3.806)$ & $<0.001$ \\
Dose of insulin at discharge & $69(12.57)$ & $36(3.806)$ & $<0.001$ \\
P-value** $^{* *}$ & $<0.001$ & $<$ & \\
\hline
\end{tabular}

*P-value for between subjects analysis; **P-value for within subjects analysis

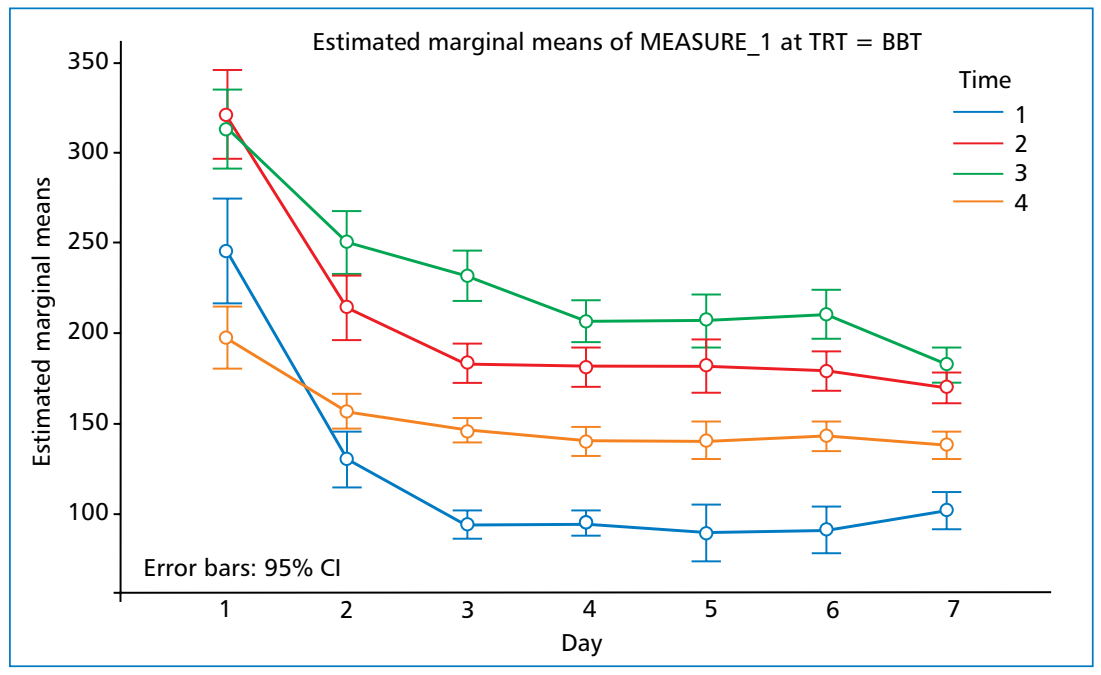

Figure 1. Longitudinal analysis of CBG from baseline to study end across 4 time points: basal-bolus therapy $(n=30)$. Measure_1: CBG

tion of two arginine residues to the C-terminus of the $\mathrm{B}$-chain. The solution of insulin glargine injection has a $\mathrm{pH}$ of 4 , which neutralises post-injection to $\mathrm{pH} 7$. The addition of arginine residues increases the isoelectric point of insulin glargine and results in formation of a microprecipitate within an amorphous SC depot, from which slow and protracted release of insulin glargine occur. Glargine-300 is a formulation of insulin glargine that delivers the same amount of insulin units as insulin glargine $100 \mathrm{U} / \mathrm{mL}$ (Gla-100) in one-third of the injection volume.

Glargine-300 comprises the same active glargine molecule as Gla-100 but forms a more compact SC depot with a reduced surface area than Gla-100. It is hypothesized that the size, and hence the surface area, of the SC depot determines the re-dissolution rate. This may allow for a longer SC residence time and degradation by tissue peptidases, resulting in a reduced 


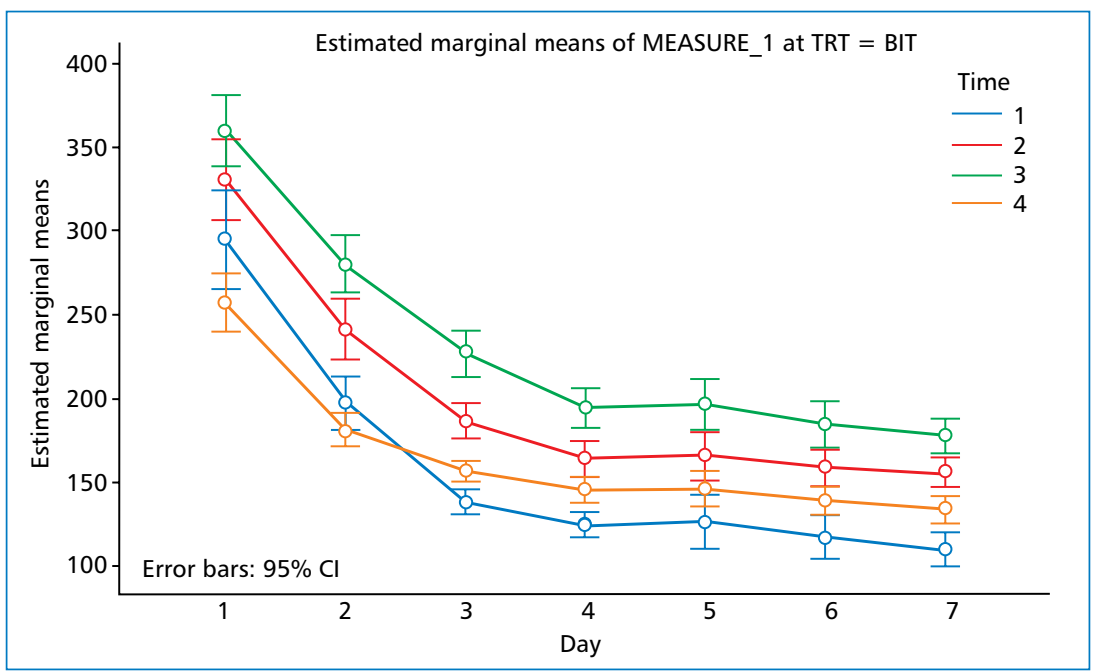

Figure 2. Longitudinal Analysis of CBG from baseline to study end across 4 time points: basal insulin therapy $(n=30)$. Measure_1: CBG. Lines 1, 2, 3 and 4 represents the time points $8 \mathrm{am}, 1 \mathrm{pm}, 7 \mathrm{pm}$ and midnight in both graphs. It represents the average capillary blood glucose (CBG) levels on each day at the stated time. TRT = BBT represents the graph for treatment of basal bolus therapy - human actrapid thrice daily as bolus and glargine U 100 as basal. TRT = BIT represents graph for basal insulin therapy using glargine u $\mathbf{3 0 0}$ mono therapy

re-dissolution rate. Accordingly, Glargine-300 has a more stable activity profile and a more prolonged and gradual insulin release than Glargine-100, resulting in blood glucose control that lasts for up to 36 hours [15].

A prospective, randomized multi-center trial compared the efficacy and safety of a with basal bolus regimen and sliding scale insulin regimen (SSI) in patients with type 2 diabetes admitted to a general medicine service [16]. The use of basal-bolus insulin had greater improvement in blood glucose control than sliding scale alone. A blood glucose target $<140 \mathrm{mg} / \mathrm{dL}$ $(7.8 \mathrm{mmol} / \mathrm{L})$ was achieved in $66 \%$ of patients in the glargine plus glulisine group and $38 \%$ in the sliding scale group. The incidence of hypoglycemia, defined as a $B G<60 \mathrm{mg} / \mathrm{dL}(3.3 \mathrm{mmol} / \mathrm{L})$, was less than $5 \%$ in patients treated with basal bolus or SSI. A different study in general surgery patients also compared efficacy and safety of a basal bolus regimen to SSI in patients with type 2 diabetes [17]. The basal bolus regimen resulted in a significant improvement in glucose control and in a reduction in the frequency of the composite of postoperative complications including wound infection, pneumonia, respiratory failure, acute renal failure and bacteremia.

Smiley et al. have done a post-hoc analysis of a prospective, multicenter, randomized trial of 298 nonICU medicine and surgery patients with T2D treated with basal-bolus regimen with glargine once daily and glulisine before meals and with basal-plus regimen with glargine once daily and supplemental doses of glulisine before meals for blood glucose (BG) > $140 \mathrm{mg} / \mathrm{dL}$.
Major study outcomes included differences in mean daily BG, frequency of treatment failures (defined as $>2$ consecutive $B G>240 \mathrm{mg} / \mathrm{dL}$ or a mean daily BG > $240 \mathrm{mg} / \mathrm{dL}$ ), and hypoglycemia between the medicine and surgery cohorts. They concluded that the basal-plus regimen with glargine once daily and correction doses with glulisine before meals resulted in similar glycemic control to basal bolus regimen. They observed no differences in response to either basal insulin regimen between medicine and surgery patients with type 2 diabetes [18]. Francisco et al. conducted a prospective, open-label, randomized clinical trial included 176 patients with poorly controlled T2D (admission blood glucose [BG] $228 \pm 82 \mathrm{mg} / \mathrm{dL}$ and $\mathrm{HbA}_{1 \mathrm{c}} 9.5 \pm 2.2 \%$ ), treated with oral agents or insulin before admission. Patients were treated with a basal-bolus regimen with glargine U300 ( $n=92$ ) or glargine U100 ( $n=84)$ and glulisine before meals. They adjusted insulin daily to a target BG of $70-180 \mathrm{mg} / \mathrm{dL}$. The primary end point was noninferiority in the mean difference in daily $B G$ between groups. The major safety outcome was the occurrence of hypoglycemia. Hospital treatment with glargine U300 resulted in similar glycemic control compared with glargine U100 and may be associated with a lower incidence of clinically significant hypoglycemia [19]. Although the above mentioned studies are not similar to our study, they have established basal insulin glargine as a cornerstone of basal-bolus or basal-plus insulin regimen.

Till date we have not found any study comparing basal insulin therapy (BIT) with basal-bolus insulin 
therapy (BBIT), even after extensive search of literature. This is the most important strength of our study. In our study, we have found there was significant difference in attaining glycemic target (140-180 mg/dL) between two groups of therapy with better glycemic control with BIT group receiving glargine $\mathrm{U} 300$ as monotherapy. However, if we try to achieve strict glycemic control (110-140 mg/dL), there was no significant difference between two groups of therapy. Number of patients experiencing hypoglycemic episodes was also less in BIT group in comparison to BBIT group. Patients treated with basal insulin by glargine U300 had less insulin requirement during admission and discharge compared to basal bolus regimen.

Glycemic variability (GV), which refers to fluctuation in in blood glucose levels, has a broader meaning because it alludes to blood glucose oscillations that occur throughout the day, including hypoglycemic periods and postprandial increases, as well as blood glucose fluctuations that occur at the same time on different days. The broad definition of GV considers the intraday glycemic excursions, including episodes of hyperglycemia and hypoglycemia. In recent years, GV has been proposed to be an additional risk factor for complications of diabetes independent of hyperglycemia . Growing evidence indicates that significant GV, particularly when accompanied by hypoglycemia, can have a harmful effect not only on the onset and progression of diabetes complications but also in clinical conditions other than diabetes treated in intensive care units (ICUs) [19]. GV is a physiological phenomenon that assumes an even more important dimension in the presence of diabetes because it not only contributes to increasing the mean blood glucose values but it also favours the development of chronic diabetes complications. It appears that GV is poised to become a future target parameter for optimum glycemic control over and above standard glycemic parameters, such as blood glucose and $\mathrm{HbA} 1 \mathrm{c}$. Avoiding both hyperglycemia and hypoglycemia by careful use of SMBG and the availability of new agents to correct hyperglycemia without inducing hypoglycemia is expected to reduce the burden of premature mortality and disabling CV events associated with diabetes mellitus [19]. We have found less intraday and interday glycemic variability in patients treated with basal insulin glargine U300 compared to basal bolus therapy with glargine U100 and regular human insulin.

Limitations of this study are:

- small sample size;

- single centered study;

- use of only basal insulin therapy may not be applicable in that part of world where patients ad- mitted in hospital ward are allowed to take food from outside;

- lack of post prandial glucose measurements which is expected in basal insulin therapy group.

\section{Conclusion}

Basal-bolus insulin regimen is an established method of glycemic control in non ICU setting among hospitalised patients. Basal only insulin therapy using glargine U300 is non-inferior to basal bolus insulin to achieve glycemic target as laid by ADA in non-critically ill hospitalized patients. It also has less number of hypoglycemia, less insulin requirement and less glycemic variability. However, there is no statistically significant difference if we want to achieve strict glycemic control as critically ill patients. There is need for further randomised controlled trial to establish the efficacy and safety of Glargine U300 as monotherapy as basal insulin for in hospital diabetes care with continuous glucose monitoring method.

\section{Acknowledgements}

The authors would like to thank Medclin Research, Kolkata for their support in manuscript and statistical analysis.

\section{Conflict of interest}

None declared.

\section{REFERENCES}

1. Umpierrez GE, Smiley D, Zisman A, et al. Randomized study of basal-bolus insulin therapy in the inpatient management of patients with type 2 diabetes (RABBIT 2 trial). Diabetes Care. 2007; 30(9): 2181-2186, doi: 10.2337/dc07-0295, indexed in Pubmed: 17513708.

2. American Diabetes Association. Economic Costs of Diabetes in the U.S. American Diabetes Association Diabetes Care . 2018; 41(5): 917-928, doi: 10.2337/dci18-0007.

3. Frisch A, Chandra P, Smiley D, et al. Prevalence and clinical outcome of hyperglycemia in the perioperative period in noncardiac surgery. Diabetes Care. 2010; 33(8): 1783-1788, doi: 10.2337/ dc10-0304, indexed in Pubmed: 20435798.

4. Pasquel FJ, Spiegelman R, McCauley M, et al. Hyperglycemia during total parenteral nutrition: an important marker of poor outcome and mortality in hospitalized patients. Diabetes Care. 2010; 33(4): 739-741, doi: 10.2337/dc09-1748, indexed in Pubmed: 20040658.

5. Davidson P, Kwiatkowski CA, Wien M. Management of Hyperglycemia and Enteral Nutrition in the Hospitalized Patient. Nutr Clin Pract. 2015; 30(5): 652-659, doi: 10.1177/0884533615591057, indexed in Pubmed: 26084507.

6. Pleva M, Mirtallo JM, Steinberg SM. Hyperglycemic events in nonintensive care unit patients receiving parenteral nutrition. Nutr Clin Pract. 2009; 24(5): 626-634, doi: 10.1177/0884533609339069, indexed in Pubmed: 19564627.

7. Pancorbo-Hidalgo PL, García-Fernandez FP, Ramírez-Pérez C. Complications associated with enteral nutrition by nasogastric tube in an internal medicine unit. J Clin Nurs. 2001; 10(4): 482-490, doi: 10.1046/j.1365-2702.2001.00498.x, indexed in Pubmed: 11822496. 
8. McCowen K, Malhotra A, Bistrian B. Stress-Induced hyperglycemia. Critical Care Clinics. 2001; 17(1): 107-124, doi: 10.1016/ s0749-0704(05)70154-8.

9. ANDREWS R, WALKER B. Glucocorticoids and insulin resistance: old hormones, new targets. Clinical Science. 1999; 96(5): 513, doi: $10.1042 / \operatorname{cs} 19980388$.

10. Marathe CS, Rayner CK, Bound M, et al. Small intestinal glucose exposure determines the magnitude of the incretin effect in health and type 2 diabetes. Diabetes. 2014; 63(8): 2668-2675, doi: 10.2337/db13-1757, indexed in Pubmed: 24696447.

11. Korytkowski MT, Salata RJ, Koerbel GL, et al. Insulin therapy and glycemic control in hospitalized patients with diabetes during enteral nutrition therapy: a randomized controlled clinical trial. Diabetes Care. 2009; 32(4): 594-596, doi: 10.2337/dc08-1436, indexed in Pubmed: 19336639.

12. Cook CB, Castro JC, Schmidt RE, et al. Diabetes care in hospitalized noncritically ill patients: More evidence for clinical inertia and negative therapeutic momentum. J Hosp Med. 2007; 2(4): 203-211, doi: 10.1002/jhm.188, indexed in Pubmed: 17683100.

13. American Diabetes Association. Diabetes Care in the Hospital. Diabetes Care. 2020; 43(Suppl 1): S193-S202, doi: 10.2337/ dc20-S015, indexed in Pubmed: 31862758.

14. Pasquel F, Lansang $M$, Khowaja A, et al. A randomized controlled trial comparing glargine U300 and glargine U100 for the inpatient management of medicine and surgery patients with type 2 diabetes: glargine U300 hospital trial. , doi: 10.2337/dc20-1234/ suppl.12016428.v1.

15. Ghosh S, Ghosh R. Glargine-300: An updated literature review on randomized controlled trials and real-world studies. World J Diabetes. 2020; 11(4): 100-114, doi: 10.4239/wjd.v11.i4.100, indexed in Pubmed: 32313609.

16. Umpierrez GE, Smiley D, Jacobs $S$, et al. Randomized study of basal-bolus insulin therapy in the inpatient management of patients with type 2 diabetes (RABBIT 2 trial). Diabetes Care. 2007; 30(9): 2181-2186, doi: 10.2337/dc07-0295, indexed in Pubmed: 17513708.

17. Umpierrez GE, Smiley D, Jacobs $S$, et al. Randomized study of basal-bolus insulin therapy in the inpatient management of patients with type 2 diabetes undergoing general surgery (RABBIT 2 surgery). Diabetes Care. 2011; 34(2): 256-261, doi: 10.2337/ dc10-1407, indexed in Pubmed: 21228246.

18. Smiley D, Umpierrez GE, Hermayer K, et al. Differences in inpatient glycemic control and response to subcutaneous insulin therapy between medicine and surgery patients with type 2 diabetes. Diabetes Complications. 2013; 27(6): 637-641, doi: 10.1016/j. jdiacomp.2013.05.007, indexed in Pubmed: 23911535.

19. Suh S, Kim JH. Glycemic variability: how do we measure it and why is it important? Diabetes Metab J. 2015; 39(4): 273-282, doi: 10.4093/dmj.2015.39.4.273, indexed in Pubmed: 26301188 